ELECTRONIC RESEARCH ANNOUNCEMENTS OF THE AMERICAN MATHEMATICAL SOCIETY

Volume 9, Pages 135-141 (December 17, 2003)

S $1079-6762(03) 00120-3$

\title{
HARMONIC FUNCTIONS ON ALEXANDROV SPACES AND THEIR APPLICATIONS
}

\author{
ANTON PETRUNIN
}

(Communicated by Dmitri Burago)

\begin{abstract}
The main result can be stated roughly as follows: Let $M$ be an Alexandrov space, $\Omega \subset M$ an open domain and $f: \Omega \rightarrow \mathbb{R}$ a harmonic function. Then $f$ is Lipschitz on any compact subset of $\Omega$.

Using this result I extend proofs of some classical theorems in Riemannian geometry to Alexandrov spaces.
\end{abstract}

\section{INTRODUCTION}

Most of the work presented in this paper was done seven years ago; here, I publish only the announcement of the results, since the proofs are very technical. For complete proofs I refer the reader to Pet1].

During the years since most of the work was done, there have been some developments in the field, mostly due to Kuwae, Machigashira and Shioya [KMS1, KMS2. The reader is referred to their papers for the updates.

In this paper, I am trying to establish basic properties of harmonic functions defined on an Alexandrov space. As an application I obtain generalizations of the Isoperimetric Inequality of Gromov-Levy type and extend the standard estimates of eigenvalues to Alexandrov spaces.

The main technical tool relies on the fact that a harmonic function on a space with a lower curvature bound is Lipschitz on any compact subdomain. More precisely, if $M$ is an Alexandrov space with a lower curvature bound, $F: U \subset M \rightarrow \mathbb{R}^{n}$ is a harmonic map, and $C \subset U$ is compact, then $\left.F\right|_{C}$ is Lipschitz. In a certain sense this statement is an addition to the Gromov-Shoen Theorem, which says that any harmonic map $\mathbb{R}^{n} \rightarrow N$, where $N$ is a negatively curved (singular) space, is Lipschitz. But this addition is not so general as it should be. There is a strong feeling that the statement should be true in the case of a lower Ricci curvature bound, not just for spaces with a lower sectional curvature bound. Unfortunately, before proving this generalization, one has to define generalized spaces with a lower Ricci bound. The technique presented below could provide help in making the right definition of such spaces.

Received by the editors March 4, 2003.

2000 Mathematics Subject Classification. Primary 51K10; Secondary 31 B99.

The main part of this paper was written while I had postdoctoral fellowship at MSRI in 1995-1996. I would like to thank this institute for providing excellent conditions to conduct this research. I was also supported by NSF DMS-0103957. 
The original motivation for investigating harmonic functions was to get a local analysis, which is desperately needed for Alexandrov spaces (for example, to prove that the boundary of an Alexandrov space is an Alexandrov space). This motivation seemed to be reasonable, since harmonic functions already give a way to establish a good analysis on both Alexandrov spaces with two-sided bounded curvature (see [N]) and on 2-dimensional Alexandrov spaces with bounded curvature (in particular, for curvature bounded below) (see $[\mathrm{R}$ ). Unfortunately this idea encountered an apparently small obstacle: I could not construct a harmonic chart in a neighborhood of any point of an Alexandrov space (even a "very good" point).

\section{List OF RESUlTS AND SOME DISCUSSION}

1.A. Main Theorem. In an Alexandrov $n$-space, the space of Sobolev functions can be understood as the closure of Lipschitz functions under the following norm:

$$
\|f\|_{1,2}^{2}=\int_{\Omega}\left(f^{2}+|\operatorname{grad} f|^{2}\right) d h_{n}
$$

where $h_{n}$ is Hausdorff measure and $|\operatorname{grad} f|$ is the maximal rate of growth of $f$ at a point. This norm makes it possible to define a $\lambda$-harmonic function as the energy minimizing function having fixed boundary values, where the energy of a function $f$ is given by

$$
E_{\lambda}(f)=\int_{\Omega}\left(\lambda f+|\operatorname{grad} f|^{2}\right) d h_{n} .
$$

The $\lambda$-subharmonic ( $\lambda$-superharmonic) functions could be defined in the same way, but $f$ is supposed to minimize the energy of all functions $\leq f(\geq f)$ with the same boundary values.

The main result of this paper can now be formulated:

Theorem A. Let $\Omega$ be an open domain in an Alexandrov space $M$ with curvature $\geq k$, and let $C \subset \Omega$ be a compact subset of diameter $\leq D$ and volume $\geq v$. Then there is a constant $L=L(D, v, k, \operatorname{dist}(\partial \Omega, C))$ such that if $f: \Omega \rightarrow \mathbb{R}$ is a harmonic function, then $\left.f\right|_{C}$ is a Lipschitz function with Lipschitz constant $L\|f\|_{1,2}$.

1.B. Properties of harmonic functions. The next theorem says, roughly, that the limit of harmonic functions on an Alexandrov space is again a harmonic function.

Theorem B. Let $\left\{M_{i}\right\}$ be a noncollapsing sequence of Alexandrov spaces, and let $M$ be its Gromov-Hausdorff limit $\left(\operatorname{dim} M=\operatorname{dim} M_{i}\right)$. Let $f_{i}: B_{R}\left(p_{i}\right) \subset M_{i} \rightarrow \mathbb{R}$ be a sequence of harmonic functions with a uniformly bounded norm and $f: B_{R}(p) \subset$ $M \rightarrow R$ its limit. Then $f$ is a harmonic function and for any $R^{\prime}<R$

$$
\left\|\left.f\right|_{B_{R^{\prime}}(p)}\right\|_{1,2}=\lim _{i \rightarrow \infty}\left\|\left.f_{i}\right|_{B_{R^{\prime}}\left(p_{i}\right)}\right\|_{1,2} \text {. }
$$

Using this theorem one can define the differential $d_{p} f: C_{p} \rightarrow \mathbb{R}$ of a harmonic function $f$ as the limit of $\lambda_{i}(f-f(p))$ on the blown up space $\left(\lambda_{i} M, p\right)$, for $\lambda_{i} \rightarrow \infty$. It is not known whether this construction depends on the choice of the sequence $\left\{\lambda_{i}\right\}$. However, in view of Theorem A there are converging subsequences, and by Theorem B the limit function $d_{p} f: C_{p} \rightarrow \mathbb{R}$ is a harmonic function. Moreover it is easy to see that $d_{p} f$ is linear, i.e., there is a geometrical splitting $C_{p}=\mathbb{R} \times L_{p}$, where $L_{p}$ is a cone with nonnegative curvature, such that if $C_{p} \ni x=\left(x_{\mathbb{R}}, x_{L}\right)$, then $d_{p} f(x)=c x_{\mathbb{R}}$. The set of linear functions on $C_{p}$ forms a vector space (of 
dimension generally $\leq n$ ), and in particular one can define the scalar product for these functions in the usual way. Moreover, using Theorem A, it is not hard to show that the constant $c$ does not depend on the sequence $\left\{\lambda_{i}\right\}$.

The last assertion gives the following corollary:

Corollary. For any two harmonic functions $f$ and $g$, the number $\left\langle d_{p} f, d_{p} g\right\rangle$ is uniquely defined at each point $p \in M$ (i.e., $\left\langle d_{p} f, d_{p} g\right\rangle$ does not depend on the rescaling sequence).

(It is possible to define a norm of $d_{p} f$ for any harmonic function $f$, but the direction formally could rotate depending on the sequence of rescaling. The corollary shows that directions of the differential rotate the same way for all harmonic functions defined at this point.)

The Corollary gives more support to the idea that harmonic functions could give more useful charts for Alexandrov spaces.

1.C. Gromov-Levy Isoperimetric Inequality. The following generalization of the Gromov-Levy Isoperimetric Inequality is supposed to be proven for a generalized space with Ricc $\geq n-1$. Unfortunately defining such spaces in a reasonable way is difficult.

Theorem C. Let $\Sigma^{n}$ be an Alexandrov space with curvature $\geq 1$ and $\sigma_{\alpha}$ a surface which divides the volume of $\Sigma^{n}$ in ratio $\alpha$. Let $S^{n}$ be a standard sphere and $s_{\alpha}$ a sphere in $S^{n}$ which divides the volume of $S^{n}$ in the same ratio $\alpha$. Then

$$
\frac{\operatorname{vol}\left(\sigma_{\alpha}\right)}{\operatorname{vol}\left(s_{\alpha}\right)} \geq \frac{\operatorname{vol}\left(\Sigma^{n}\right)}{\operatorname{vol}\left(S^{n}\right)}
$$

and if the inequality is exact, then $\Sigma^{n}=S\left(\Lambda^{n-1}\right)$ (a geometric spherical suspension over another Alexandrov space $\Lambda^{n-1}$ with curvature $\left.\geq 1\right)$.

1.D. Estimate of eigenvalues of Alexandrov space. Finally, I present a simple application of Theorem A.

Theorem D. The first eigenvalue $\lambda_{1}$ of an $n$-dimensional Alexandrov space with curvature $\geq 1$ is at least $n$.

1.E. Remarks about the proofs. I now present some remarks about the proofs, which involve many technical details.

On the proof of Theorem A. A complete proof of Theorem A is contained in Pet1. For simplicity, I will consider only the case of nonnegative curvature (i.e. $k \geq 0$ ).

First, from [KMS1], harmonic functions are bounded on any compact subdomain (my original seven-year-old proof of this part was more complicated). It would be enough to prove that the absolute value of the gradient of a harmonic function is subharmonic. If so, the absolute value of the gradient is bounded on any compact subdomain, and therefore the original function is Lipschitz on this subdomain.

For a given function $f$, construct a one-parameter family of functions $f_{t}$; this construction resembles equidistant surfaces. Namely, let $\phi: \mathbb{R}_{+} \rightarrow \mathbb{R}_{+}$be an increasing convex $C^{\infty}$ function. Set

$$
f_{t}: \Omega_{t} \rightarrow \mathbb{R}, \quad f_{t}(x)=\max _{y \in \Omega} f(y)-t \phi\left(\frac{|x y|}{t}\right),
$$

where $\Omega_{t}$ is the maximal domain of $x$ such that the maximum $\max _{y \in \Omega} f(y)-t \phi\left(\frac{|x y|}{t}\right)$ is not admitted on the boundary of $\Omega$. Note that $f_{t+\tau}=\left(f_{t}\right)_{\tau}$ and $\Omega_{t+\tau}=\left(\Omega_{t}\right)_{\tau}$. 
Note also that if $\phi$ grows rapidly enough, say $\phi(x)=x^{2}$, then the theorem above yields that, for any compact $C \subset \Omega$, there is a subdomain $\Omega^{\prime}$ such that $C \subset \Omega^{\prime} \subset \Omega$ and $C \subset \Omega_{t}^{\prime}$ for small enough $t$.

Instead of proving that the absolute value of the gradient is subharmonic, I prove a weaker result:

Key Lemma. Let $f: \Omega \rightarrow \mathbb{R}$ be $\lambda$-subharmonic. Then $f_{t}: \Omega_{t} \rightarrow \mathbb{R}$ is a $\lambda$ subharmonic function.

Although the Key Lemma seems obvious, its proof is technically very difficult, and I refer the reader to Pet1] for the complete proof.

Now consider functions $\left(f_{t}-f\right) / t$. Since $f$ is harmonic, these functions are positive subharmonic functions. It is not hard to see that these functions are bounded in the $L^{2}$-norm in any $C \subset \Omega$. (To see this, note that since $f_{t}$ subharmonic, we get an estimate for $\int\left|\operatorname{grad}\left(f_{t}-f\right) / t\right|^{2}$, which gives us an estimate for $\int\left|\left(f_{t}-f\right) / t\right|^{2}$.) Since harmonic functions are bounded on $C$, I have an estimate of $\max \left(f_{t}-f\right) / t$ for any $t$ and $x \in C^{\prime}$ inside of $C$. Note that $\lim \left(f_{t}-f\right) / t$ gives an estimate of $|\operatorname{grad} f|$. It shows, in particular, that $|\operatorname{grad} f|$ is bounded on $C$, and therefore $f$ is Lipschitz. After a little extra work, one can prove that in fact $|\operatorname{grad} f|$ is subharmonic.

On the proof of Theorem $B$. Theorem A gives that $f$ is Lipschitz on any $B_{R^{\prime}}(p)$. If $f$ does not minimize energy, then one can construct a function $g$ on $B_{R^{\prime}}(p)$ with the same boundary values, with smaller energy, which is still Lipschitz, and such that it is different from $f$ only on $B_{R^{\prime \prime}}(p), R^{\prime \prime}<R^{\prime}$. Using the distance charts one can easily lift $g$ to $g_{i}$ on $M_{i}$ in such a way that $g_{i}$ will have approximately the same $\|*\|_{1,2^{-}}$ norm as $g$. Take a nice Lipschitz function $\lambda: \mathbb{R}_{+} \rightarrow[0,1]$ which is 1 for $x \leq R^{\prime \prime}$ and 0 for $x \geq R^{\prime}$; mix the functions $f_{i}$ and $g_{i}$ by $\lambda\left(\left|p_{i} x\right|\right) g_{i}(x)+\left(1-\lambda\left(\left|p_{i} x\right|\right)\right) f_{i}(x)$. Thus, for sufficiently large $i$, we get a function with smaller energy. But the functions $f_{i}$ are harmonic, a contradiction.

The rest of the properties are trivial corollaries of the above construction and Theorem B by itself.

On the proof of Theorem C. The original proof of the Gromov-Levy inequality (see G]) uses the smoothness of minimal surfaces. It refers to a theorem of Almgren A, which is not trivial on its own, but simply cannot be true in a nonsmooth space (at least directly). The proof of Theorem $\mathrm{C}$ proceeds along the same lines as those in $\mathrm{G}$ but avoids the use of Almgren's result.

A good thing about the following proof is that it does not use smoothness at all; it only uses the existence. One can get the existence as a direct application of the classical theory of currents (see $[\mathrm{F}]$ ).

Let $\sigma_{\alpha}$ be the minimal set which divides an Alexandrov space $\Sigma$ in ratio $\alpha$, and let $\Sigma_{+}$and $\Sigma_{-}$be the two connected components of $\Sigma \backslash \sigma$. Without loss of generality I can assume that for each point $x \in \sigma_{\alpha}$ and $\epsilon>0$, the volume $\operatorname{vol}\left(\Sigma_{ \pm} \cap B_{\epsilon}(x)\right)>0$.

Consider the functions $f_{+}: \Sigma_{+} \rightarrow \mathbb{R}_{+}$and $f_{-}: \Sigma_{-} \rightarrow \mathbb{R}_{+}, f_{ \pm}=d i s t_{\sigma_{\alpha}}$.

Now let $s^{*}$ be a sphere in $S^{n}$. One defines $S_{+}^{n}, S_{-}^{n}, f_{+}^{*}: S_{+}^{n} \rightarrow \mathbb{R}$ and $f_{-}^{*}: S_{-}^{n} \rightarrow \mathbb{R}$ in the same way as above.

The following lemma should be regarded as an analog of the fact that a minimal surface has constant mean curvature. 
Lemma. There is a sphere $s^{*}$ in $S^{n}$ such that if $f_{ \pm}^{*}(y)=f_{ \pm}(x)$, then $\Delta f_{ \pm}^{*}(y) \geq$ $\Delta f_{ \pm}(x)$.

The last inequality should be understood as an estimate for $\Delta f_{ \pm}$, i.e., for any $\epsilon>0$ and any $x \in \Sigma_{ \pm}$, there is a neighborhood $U \ni x$ such that $f_{ \pm}$is a $\left(\Delta f_{ \pm}^{*}(y)-\epsilon\right)$ superharmonic function in $U$.

It is easy to construct two functions $g_{ \pm}$defined in a neighborhood of $x_{ \pm}$, which support $f_{ \pm}$at these points (i.e. $g_{ \pm}(x) \leq f_{ \pm}(x)$ and the inequality is exact only if $\left.x=x_{ \pm}\right)$, and such that $\Delta g_{ \pm} \geq \Delta f_{ \pm}\left(y_{ \pm}\right)+\epsilon$. One can assume that the foot points of $x_{+}$and $x_{-}$(say $z_{+}$and $z_{-}$) on $\sigma_{\alpha}$ are distinct.

Consider the functions $\tilde{g}_{ \pm}(y)=\max g_{ \pm}(x)-|x y|$. These functions $\tilde{g}_{ \pm}(y)$ are well defined in a neighborhood of the minimal geodesic $x_{ \pm} z_{ \pm}$, i.e. the minimum is admitted for interior points of the domain of $g_{ \pm}$.

As a corollary of the Key Lemma (for Theorem A), it is easy to see that $\tilde{g}_{ \pm}$ are $\left(\Delta f_{ \pm}\left(y_{ \pm}\right)+\epsilon\right)$-subharmonic. Moreover, direct calculations (analogous to the Rauch comparison for Riemannian manifolds) show that in some neighborhoods of $z_{ \pm}$these functions are $\lambda_{ \pm}$-subharmonic with $\lambda_{+}+\lambda_{-}>0$.

The "direct calculations" above for the general case use the second variation formula for Alexandrov spaces [Pet2] and Perelman's representation of a semiconvex function. The latter says that for any semiconvex function $f$ one has the following representation for almost all $x$ :

$$
f(y)=f(x)+\left\langle\operatorname{grad} f, \log _{x} y\right\rangle+H\left(\log _{x} y\right)+o\left(|x y|^{2}\right),
$$

where $H$ is a quadratic form, $\log _{x} y$ is an element of the tangent space $C_{x}$ at $x$ such that $\left|\log _{x} y\right|=|x y|$, and the direction of $\log _{x} y$ coincides with the direction from $x$ to $y$.

The second variation gives an estimate of $H$. The regular part of $\Delta f$ is simply trace $(H)$, and the singular part is positive since the function is semiconvex.

Let me cut two small caps with the same volume along level sets of these functions (Figure 1). Let $A_{+o l d}, A_{-o l d}$ be the areas of these caps and $A_{+n e w}, A_{-n e w}$ the areas of the level sets which cut these caps. Let $v=v_{+}=v_{-}$be the volume being cut. Then from the Stokes theorem,

$$
A_{ \pm \text {new }}-A_{ \pm \text {old }} \leq \lambda_{ \pm} v .
$$

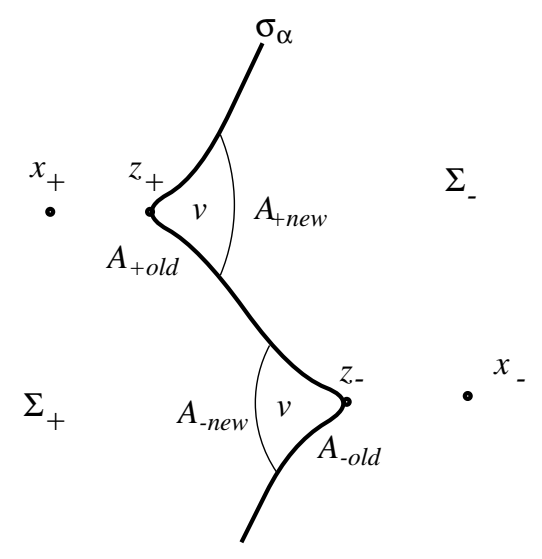

FiguRE 1. 
Therefore

$$
A_{+ \text {new }}+A_{- \text {new }}<A_{+ \text {old }}+A_{- \text {old }} .
$$

Thus, the area of $\sigma_{\alpha}$ decreases, which is a contradiction, since $\sigma_{\alpha}$ is already minimal.

The rest of the proof of Theorem $\mathrm{C}$ is only a small variation of the original one. From the inequality in the lemma, one has the differential inequality for $a_{ \pm}(r)=\operatorname{Area}\left(\sigma_{r \pm}\right)$ (where $\sigma_{r \pm} \subset \Sigma_{ \pm}$at the distance $r$ from $\left.\sigma_{\alpha}\right)$ :

$$
a_{ \pm}^{\prime} / a_{ \pm}<a_{* \pm}^{\prime} / a_{* \pm}
$$

Now consider a round sphere $s_{\alpha}$ in $S^{n}$ which divides $S^{n}$ in the same ratio $\alpha$. Without loss of generality I can assume that $a_{*+}^{\prime} / a_{*+} \leq a_{\alpha,+}^{\prime} / a_{\alpha,+}$. Integrating this inequality, I get

$$
\frac{\operatorname{vol}\left(\Sigma_{+}\right)}{\operatorname{vol}\left(\sigma_{\alpha}\right)} \leq \frac{\operatorname{vol}\left(S_{\alpha,+}^{n}\right)}{\operatorname{vol}\left(s_{\alpha}\right)},
$$

and using that $\sigma_{\alpha}$ and $s_{\alpha}$ divide their respective spaces in the same ratio, I get

$$
\frac{\operatorname{vol}(\Sigma)}{\operatorname{vol}\left(\sigma_{\alpha}\right)} \leq \frac{\operatorname{vol}\left(S^{n}\right)}{\operatorname{vol}\left(s_{\alpha}\right)},
$$

or

$$
\frac{\operatorname{vol}\left(\sigma_{\alpha}\right)}{\operatorname{vol}\left(s_{\alpha}\right)} \geq \frac{\operatorname{vol}\left(\Sigma^{n}\right)}{\operatorname{vol}\left(S^{n}\right)} .
$$

Proof of Theorem D. Let $\phi_{1}$ be an eigenfunction with eigenvalue $\lambda_{1}$. Consider a function $f_{1}: C(\Sigma) \rightarrow \mathbb{R}, f_{1}=\rho^{\lambda_{1} / n} \phi_{1}(\sigma)$, where $\rho \in \mathbb{R}_{+}, \sigma \in \Sigma,(\rho, \sigma) \in C(\Sigma)$. Easy calculations show that $f_{1}$ is a harmonic function. By Theorem A, it is Lipschitz in a neighborhood of the center of the cone; therefore $\lambda_{1} \geq n$.

\section{Questions}

2.1. Is it true that, in a neighborhood of a regular point in an Alexandrov space, there is a harmonic chart, i.e., there is a homeomorphism $f: U \rightarrow \mathbb{R}^{n}$, $x \rightarrow\left(f_{1}(x), f_{2}(x), \ldots, f_{n}(x)\right)$ such that each $f_{k}$ is harmonic?

If the dimension is 2 , then isotropic coordinates give an affirmative answer.

Note that the fact that the tangent cone $C_{p}$ always splits in the direction of $d_{p} f$ shows that if such a coordinate system exists, then the metric tensor $g^{i j}$ must be well defined at each point, but it is degenerate at each nonregular point (i.e. this metric tensor looks a little like a sub-Riemannian metric).

2.2. Is it true that the differential of a harmonic function is uniquely defined at each point?

2.3. How do the harmonic functions in Theorem $B$ behave if the sequence of spaces is allowed to collapse?

\section{REFERENCES}

[A] Almgren, F., Existence and regularity almost everywhere of solutions to elliptic variational problem with constraints, Mem. A.M.S., 4 (1976). MR 54:8420

[BGP] Burago, Yu., Gromov, M., Perelman, G., A. D. Alexandrov spaces with curvature bounded below. (English. Russian original), Russ. Math. Surv. 47, No. 2, 1-58 (1992); translation from Usp. Mat. Nauk 47, No. 2 (284), 3-51 (1992). MR 93m:53035

[F] Federer, Herbert, Geometric measure theory. Die Grundlehren der mathematischen Wissenschaften, Band 153, Springer-Verlag New York Inc., New York, 1969, xiv+676 pp. MR 41:1976 
[G] Gromov, M., Isoperimetric inequalities in Riemannian manifolds, appendix in Milman, Vitali D., Schechtman, Gideon, Asymptotic theory of finite dimensional normed spaces, Lecture Notes in Mathematics, 1200. MR 87m:46038

[LU] Ladyzhenskaya, O. A., Uraltseva, N. N., Linear and quasilinear equations of elliptic type, "Nauka", Moscow, 1973. 576 pp. MR 58:23009

[KMS1] Kuwae, Kazuhiro, Machigashira, Yoshiroh, Shioya, Takashi, Beginning of analysis on Alexandrov spaces, Geometry and topology: Aarhus (1998), 275-284, Contemp. Math., 258, Amer. Math. Soc., Providence, RI, 2000. MR 2002g:53066

[KMS2] Kuwae, Kazuhiro, Machigashira, Yoshiroh, Shioya, Takashi, Sobolev spaces, Laplacian, and heat kernel on Alexandrov spaces, Math. Z. 238 (2001), no. 2, 269-316. MR 2002m:58052

[N] Nikolaev, I. G., Smoothness of the metric of spaces with bilaterally bounded curvature in the sense of A. D. Aleksandrov, (Russian) Sibirsk. Mat. Zh. 24 (1983), no. 2, 114-132. MR 84h:53098

[Per1] Perelman, G., DC structure on Alexandrov space, preprint available at http://www.math.psu.edu/petrunin/.

[Per2] Perelman, G., A. D. Alexandrov space with curvature bounded from below II, preprint.

[PerPet] Perelman G. Ya., Petrunin A. M., Extremal subsets in Aleksandrov spaces and the generalized Liberman theorem, Algebra i Analiz 5 (1993), no. 1, 242-256, Translation: St. Petersburg Math. J. 5 (1994), no. 1, 215-227. MR 94h:53055

[Pet1] Petrunin, A., Subharmonic functions on Alexandrov space, preprint available at http://www.math.psu.edu/petrunin/.

[Pet2] Petrunin, A., Parallel transportation for Alexandrov space with curvature bounded below, Geom. Funct. Anal. 8, No. 1, 123-148 (1998). MR 98j:53048

[R] Reshetnyak Yu. G., Two-dimensional manifolds of bounded curvature. (English. Russian original) [CA] Geometry IV. Nonregular Riemannian geometry. Encycl. Math. Sci. 70, 3-163 (1993); translation from Itogi Nauki Tekh., Ser. Sovrem. Probl. Mat., Fundam. Napravleniya 70, 7-189 (1989). MR 92b:53104

Department of Mathematics, The Pennsylvania State University, University Park, PA 16802

E-mail address: petrunin@math.psu.edu 